\title{
1.3 Octave Supercontinuum Generation in Highly Ge-doped Photonic Crystal Fiber
}

\author{
Deniz Aydin ${ }^{1}$, Thibault North ${ }^{1}$, Achille Monteville ${ }^{2}$, David Landais ${ }^{2}$ and Olivier Legoffic ${ }^{2}$, David Méchin ${ }^{2}$, \\ Camille-Sophie Brès ${ }^{1}$ \\ Photonic Systems Laboratory, Ecole Polytechnique Federale de Lausanne (EPFL), CH--1015 Lausanne, Switzerland \\ Photonics Bretagne (PERFOS), France, 11 Rue Louis de Broglie, 22300 Lannion, France \\ Authore-mail address: deniz.aydin@epfl.ch
}

\begin{abstract}
We present a flat, visible 1.3-octave supercontinuum generated in a $\mathrm{GeO}_{2}$-doped photonic crystal fiber owing to an overlap of the modulation instability and Raman gain regions resulting in efficient energy transfer to new frequency components.

OCIS codes: (320.0320) Ultrafast optics; (320.6629) Supercontinuum generation; (060.2280) Fiber design and fabrication
\end{abstract}

Supercontinua are of interest for numerous industrial applications including sensing, imaging, and frequency comb generation as well as in optical device characterization. Recently, Q-switched lasers were found to be compelling candidates for supercontinuum generation in the long-pulse regime [1-3]. Here we present supercontinuum generation between $620-1552 \mathrm{~nm}$ in a highly doped photonic crystal fiber (PCF) pumped with a passively Q-switched sub-ns microchip laser at $1064 \mathrm{~nm}$. Efficient generation is enabled even at low pump powers owing to overlapping modulation instability (MI) and Raman gain regions.

We designed a hexagonal lattice, solid-core silica matrix PCF with a $20 \% \mathrm{~mol} \mathrm{GeO}_{2}$ doped, $3 \mu \mathrm{m}$ core and strong index contrast $\left(\Delta \mathrm{n}=30 \times 10^{-3}\right)$ between the core and the cladding (Fig.1a, inset) fabricated by PERFOS. The PCF has $\mathrm{n}_{2}=4.709 \times 10^{-20} \mathrm{~m}^{2} / \mathrm{W}$ and a Raman gain of $g_{\mathrm{R}}=7.07 \times 10^{-14} \mathrm{~m} / \mathrm{W}$. We measured the zero dispersion wavelengths to be $\lambda_{\text {ZDw }}=1047 \mathrm{~nm}$ and $1050 \mathrm{~nm}$ for the two principal polarization axes. Dispersion is $6.44 \mathrm{ps} / \mathrm{km}-\mathrm{nm}$ at $1064 \mathrm{~nm}$, the attenuation $0.17 \mathrm{~dB} / \mathrm{m}$ and $\gamma=50(\mathrm{~W}-\mathrm{km})^{-1}$. In Fig.1 a the supercontinuum is generated after $1 \mathrm{~m}$ of fiber for various input powers: a flatness of $<6 \mathrm{~dB}$ over $820 \mathrm{~nm}$ extending from $620 \mathrm{~nm}$ to $1440 \mathrm{~nm}$ (Fig. 1a), and $<15 \mathrm{~dB}$ over $932 \mathrm{~nm}$ between $620 \mathrm{~nm}-1552 \mathrm{~nm}$ is observed for $\mathrm{P}_{\mathrm{ave}}=7.8 \mathrm{~mW}\left(\mathrm{P}_{\text {peak }}=670 \mathrm{~W}\right)$. This result is competitive with similar sources using longer fibers $(1.8-20 \mathrm{~m})$ and higher peak powers [4-6] in the same ns pulse regime. Notably, in Fig. 1a we start seeing supercontinuum in $1 \mathrm{~m}$ of fiber (bandwidth $>250 \mathrm{~nm}$ ) at markedly low peak powers of $98 \mathrm{~W}\left(\mathrm{P}_{\mathrm{ave}}=1.1 \mathrm{~mW}\right)$ with a $0.8 \mathrm{~ns}, 14 \mathrm{kHz}$ laser, lowest reported to our knowledge.
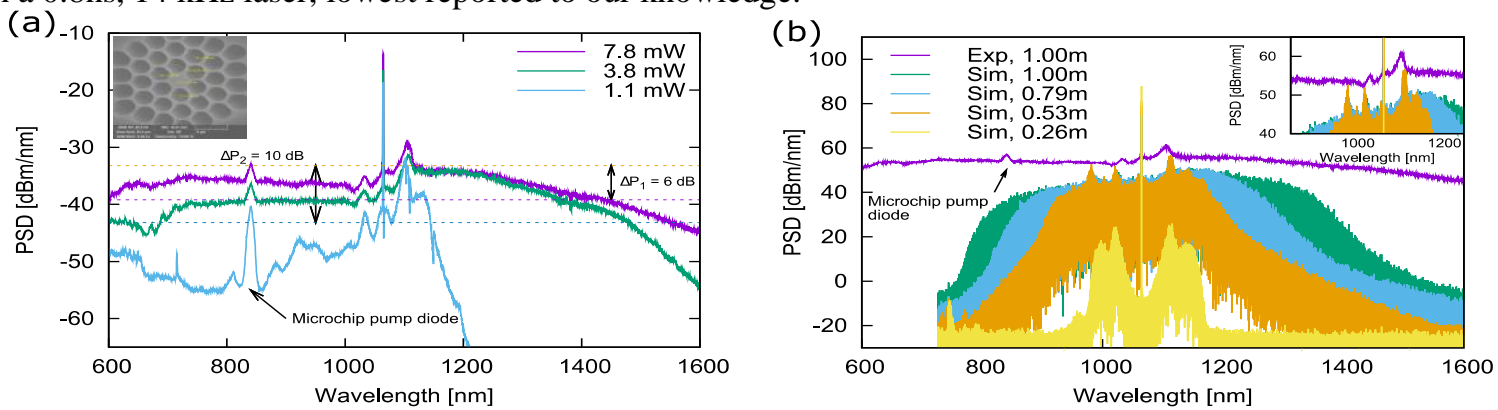

Fig, 1: (a) Experimental supercontinuum in 1m PCF, 0.8ns pulse duration at $14 \mathrm{kHz}$; (b) Simulations showing spectrum evolution as a function of fiber length and experiment in $1 \mathrm{~m}$ fiber for $\mathrm{P}_{\mathrm{ave}}=7.8 \mathrm{~mW}$, overlaid with experimental result. Traces are offset for visibility.

The generating mechanisms of this supercontinuum are studied using the generalized nonlinear Schrodinger Equation (GNLSE) solver [7], modified to include measured attenuation and quantum noise. Simulation results (Fig. 1b) show an evolution starting from MI. Raman response of $\mathrm{GeO}_{2}$ reaches its maximum at $13.2 \mathrm{THz}$ extending from $10-15 \mathrm{THz}$. Accordingly, MI and Raman peaks overlap, producing a highly efficient energy transfer from pump to shorter and longer wavelengths. This effect is primarily responsible for the extremely wideband spectrum competitive with current research-grade and commercial sources operating in the nanosecond pulse regime.

1. W. J. Wadsworth, et al., "Supercontinuum and four-wave mixing with Q-switched pulses in endlessly single-mode photonic crystal fibres," Opt. Express 12, 299-309 (2004).

2. J. M. Stone and J. C. Knight, "Visibly "white" light generation in uniform photonic crystal fiber using a microchip laser," Opt. Express 16, 2670-2675 (2008).

3.A. Mussot, et al., "20 THz-bandwidth continuous-wave fiber optical parametric amplifier operating at 1um using a dispersion-stabilized photonic crystal fiber," Opt. Express 20, 28906-28911 (2012).

4. S. Coen, et al.1, "White-light supercontinuum generation with 60-ps pump pulses in a photonic crystal fiber," Opt. Lett. 26, 1356-1358 (2001).

5. NKT Photonics, NKT Supercontinuum Application Note V2.0 July 2009 (2009).

6. C. Xiong, et al., "Enhanced visible continuum generation from a microchip 1064nm laser," Opt. Express 14, 6188-6193 (2006).

7. J. M. Dudley and S. Coen, "Supercontinuum generation in photonic crystal fiber," Rev. Mod. Phys. 78, 1135-1184 (2006). 